\title{
Construct an $f(T)$-theory from a bouncing FRW universe
}

\author{
G.G.L. Nashed ${ }^{1,2,3 *}$ and W. El Hanafy ${ }^{1,3 \dagger}$ \\ ${ }^{1}$ Centre for theoretical physics, the British University in Egypt, 11837 - P.O. Box 43, Egypt \\ ${ }^{2}$ Mathematics Department, Faculty of Science, Ain Shams University, Cairo, Egypt and \\ ${ }^{3}$ Egyptian Relativity Group (ERG)
}

\begin{abstract}
We show that the teleparallel $f(T)$ gravity can provide compatible versions of bouncing cosmologies. In this work two bounce universe models have been investigated. We present their phase portraits by constructing the phase space of the models. Also, the thermalization process of the universe during the bouncing period is discussed. We rewrite the solutions in Einstein frame to represent the torsion gravity as extra degree-offreedoms. The evolution of the equation-of-state of the torsion fluid has been studied. Assuming that the matter component to be a canonical scalar field, we obtain the scalar field potentials induced by the $f(T)$ gravity of the two suggested models. The evolution of the scalar field equation-of-state during bouncing period has been investigated. Moreover, we study the energy conditions of these two models. Finally, the slow roll validity in the bouncing cosmology is discussed.
\end{abstract}

PACS numbers: 98.80.-k, 98.80.Qc, 04.20.Cv, 98.80.Cq. Keywords: inflation, scalar field, teleparallel gravity.

doi: 10.21293/1818-0442-2016-19-4-23-33

\section{EINSTEIN'S COSMOLOGY}

Copernican (or cosmological) principle is believed to be a good approximation to construct a reliable cosmological model.

$$
d s^{2}=-d t^{2}+a(t) \delta_{i j} d x^{i} d x^{j}
$$

where $a(t)$ is the scale factor. Standard cosmology today is a manifestation of Copernican principle and Einstein's field equations of general relativity (GR) are given by,

$$
G_{\mu \nu}=\kappa^{2} \mathfrak{I}_{\mu \nu}
$$

where $G_{\mu \nu}$ is called Einstein tensor, $\kappa^{2}=8 \pi G / c^{4}, G$ is the Newtonian's gravitational constant and $c$ is the speed of light in vacuum. We assume the natural unit system $c=\hbar=k_{B}=1$, and the stress-energy tensor $\mathfrak{I}_{\mu \nu}$ is taken for a perfect fluid as,

$$
\mathfrak{I}_{\mu v}=\rho u_{\mu} u_{v}+p\left(u_{\mu} u_{v}-g_{\mu v}\right),
$$

where $u_{\mu}=\delta_{\mu}^{0}$ is the 4-velocity of the fluid in co-moving coordinates, $\rho$ and $p$ are the density and pressure of the fluid, respectively.

Applying the Einstein field equations to the FRW universe leads to Friedmann equations

$$
H^{2}=\frac{\kappa^{2}}{3} \rho, \quad \dot{H}+H^{2}=-\frac{\kappa^{2}}{6}(\rho+3 p),
$$

where $H \equiv \dot{a} / a$ is the Hubble parameter and the dot denotes the derivative with respect to time. Constraining the Friedmann equations by the equation-of-state $p=\omega \rho$, then solve for the scale factor [1]

$$
a_{\mathrm{FRW}}= \begin{cases}a_{k}\left[(1+\omega)\left(t-t_{i}\right)\right]^{\frac{2}{3(1+\omega)}}, & \omega \neq-1 ; \\ e^{H_{0}\left(t-t_{i}\right)}, & \omega=-1 .\end{cases}
$$

Where $a_{k}, H_{0}$ and $t_{i}$ are constants. The former is the usual power-law scale factor, for $\omega>-1 / 3$ the universe is expanding with deceleration, while it is accelerated when $\omega<-1 / 3$. The later gives de Sitter universe, where $\omega=-1$, which does not allow the universe to evolve, that could be considered in late phases rather inflation.

$$
\dot{H}=-\frac{3}{2}(1+\omega) H^{2}
$$

We classify the four different regions in Figure 1 as follows: (I)-region represents an accelerated contracting universe as $q<0$ and $H<0$,

(II)-region represents a decelerated contacting universe as $q>0$ and $H<0$,

(III)-region represents a decelerated expanding universe as $q>0$ and $H>0$ which characterizes the standard FRW models,

(IV)-region represents an accelerated expanding universe as $q<0$ and $H>0$ which characterizes the so-called inflation or dark energy phases.

According to the GR phase portraits, it is clear that different choice of an equation-of-state produces a different phase portrait, such that $\omega_{m}>-1 / 3$ gives decelerated phases, $\omega_{m}=$ $-1 / 3$ identifies the zero acceleration curve, while $\omega_{m}<-1 / 3$ gives accelerated phases as shown in Figure 1. Figure 2 is the phase portrait of GR plus a cosmological constant, $\Lambda$. From Fig. 2, it is clear that when $\Lambda=0$ we return to the phase portrait of Fig. 1 and when $\Lambda>0$ we have the blue curve which represents a big bang singularity followed by an inflation then a bounce after crossing the fixed point (stable one) in finite time. Same discussion can be applied when $\Lambda<0$, which represented by the red curve.

\footnotetext{
*Electronic address: nashed@bue.edu.eg

$\dagger$ Electronic address: waleed.elhanafy@bue.edu.eg
} 


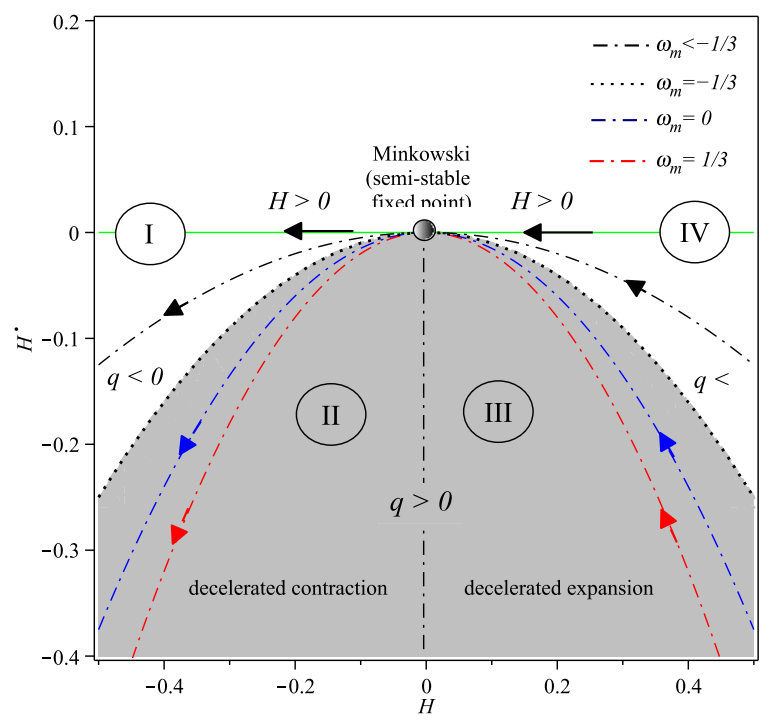

FIG. 1: The standard general relativistic phase portraits. The dot curve represents the zero acceleration boundary, it divided the phase space into two regions. The shaded region is the deceleration region, while the unshaded is the acceleration one. The labels (I)-(IV) give four possible behaviors.

We investigate the possibility to cross from $\dot{H}>0$ branch to $\dot{H}<0$ through the de Sitter fixed point $H_{2}$. The former branch goes effectively as a phantom-like $\left(\omega_{\text {eff }}<-1\right)$, while the latter is a non-phantom $\left(\omega_{e f f}>-1\right)$. The conditions for this transition to occur are listed as follows The conditions for this transition to occur are listed as follows [?]

(i) $\lim _{H \rightarrow H_{\text {inf }}} \dot{H}_{+}=0$,

(ii) $\lim _{H \rightarrow H_{\text {inf }}} d \dot{H}_{+} / d H= \pm \infty$,

(iii) $t=\int_{H}^{H_{\text {inf }}} d H / \dot{H}_{+}<\infty$.

In addition to these conditions, it has been shown that the crossing is possible only when $\dot{H}(H)$ is a doubled valued function $[2,3]$.

If we want to reproduce the above phase portraits of Fig. 3 or Fig. 4 within GR using linear equation-of-state we will get a conflict with continuity equation [3]. Therefore, we need to modify GR.

\section{PHASE PORTRAITS OF $f(T)$ COSMOLOGY}

\section{A. Teleparallel space}

This space is denoted in the literature by many names teleparallel, distant parallelism, Weitzenöck, absolute parallelism, vielbein, parallelizable space. An AP-space is a pair $\left(M, h_{a}\right)$, where $M$ is an $n$-dimensional smooth manifold and $h_{a}(a=1, \cdots, n)$ are $n$ independent vector fields defined globally on $M$. The vector fields $h_{a}$ are called the parallelization vector fields.

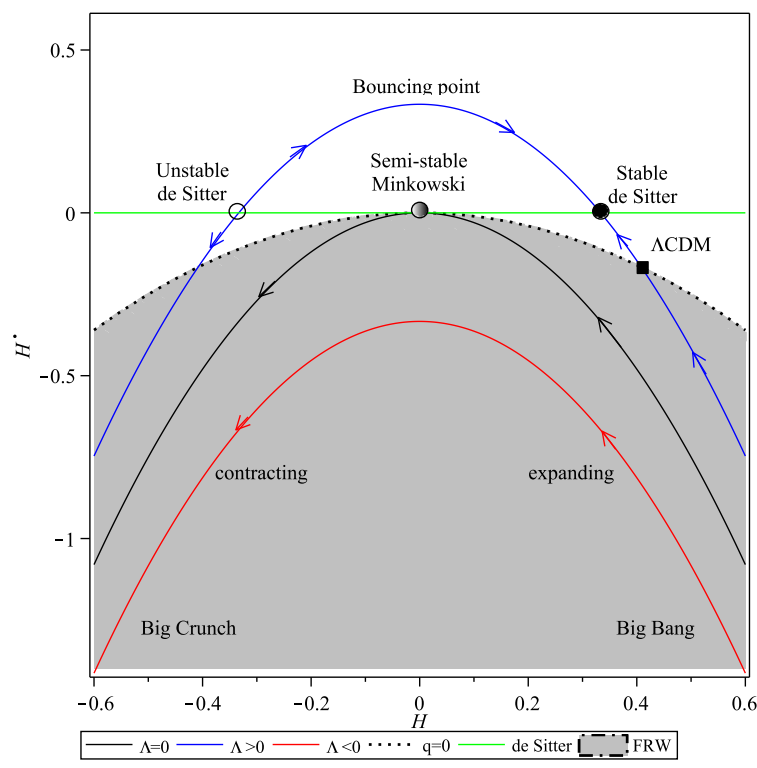

FIG. 2: $\Lambda \mathrm{CDM}$ phase portrait.

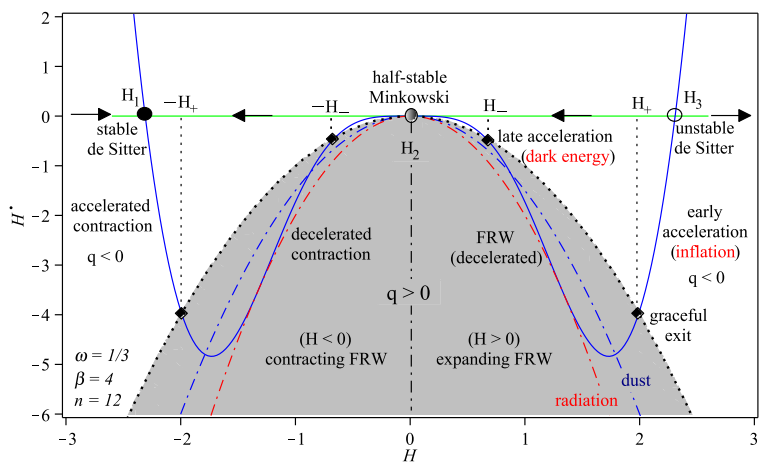

FIG. 3: From acceleration to deceleration.

Let $h_{a}{ }^{\mu}(\mu=1, \ldots, n)$ be the coordinate components of the $a$-th vector field $h_{a}$, where Greek and Latin indices are constrained by the Einstein summation convention. The covariant components $h_{a \mu}$ of $h_{a}$ are given via the relations

$$
h_{a}{ }^{\mu} h^{a}{ }_{v}=\delta_{v}^{\mu} \quad \text { and } \quad h_{a}{ }^{\mu} h^{b}{ }_{\mu}=\delta_{a}^{b},
$$

where $\delta$ is the Kronecker tensor. Because of the independence of $h_{a}$, the determinant $h \equiv \operatorname{det}\left(h_{a}{ }^{\mu}\right)$ is nonzero. On a teleparallel space $\left(M, h_{a}\right)$, there exists a unique linear connection, namely Weitzenböck connection, with respect to which the parallelization vector fields $h_{a}$ are parallel. This connection is given by

$$
\Gamma_{\mu \nu}^{\alpha} \equiv h_{a}^{\alpha} \partial_{\nu} h^{a}{ }_{\mu}=-h^{a}{ }_{\mu} \partial_{\nu} h_{a}^{\alpha},
$$

and is characterized by the property that

$$
\nabla_{v}^{(\Gamma)} h_{a}{ }^{\mu} \equiv \partial_{v} h_{a}{ }^{\mu}+\Gamma_{\lambda v}^{\mu} h_{a}{ }^{\lambda} \equiv 0,
$$

where the operator $\nabla_{v}^{(\Gamma)}$ is the covariant derivative with respect to the Weitzenböck connection. The connection (8) will also 


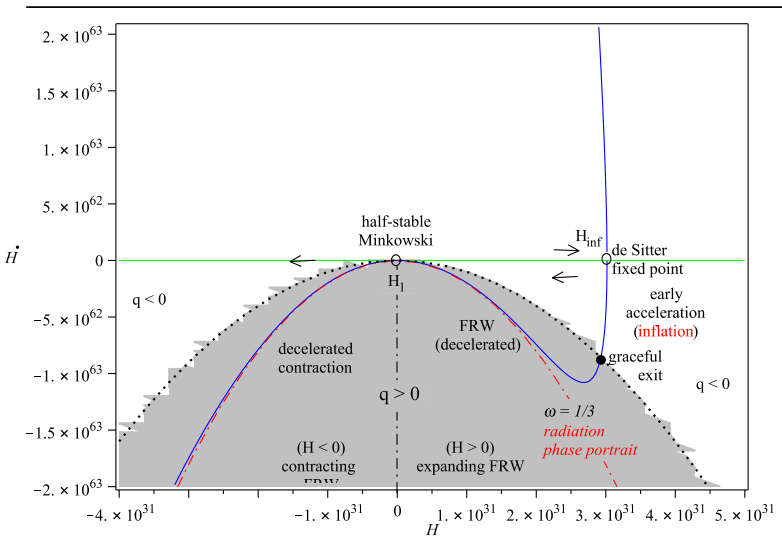

FIG. 4: Phantom cross conditions.

be referred to as the canonical connection. The relation (9) is known in the literature as the AP-condition.

The non-commutation of an arbitrary vector fields $V_{a}$ is given by

$$
\nabla_{v}^{(\Gamma)} \nabla_{\mu}^{(\Gamma)} V_{a}^{\alpha}-\nabla_{\mu}^{(\Gamma)} \nabla_{v}^{(\Gamma)} V_{a}^{\alpha}=R_{\epsilon \mu \nu}^{\alpha} V_{a}^{\epsilon}+T_{\nu \mu}^{\epsilon} \nabla_{\epsilon}^{(\Gamma)} V_{a}^{\alpha},
$$

where $R^{\alpha}{ }_{\epsilon \mu \nu}$ and $T^{\epsilon}{ }_{\nu \mu}$ are the curvature and the torsion tensors of the canonical connection, respectively. The AP-condition (9) together with the above non-commutation formula force the curvature tensor $R^{\alpha}{ }_{\mu \nu \sigma}$ of the canonical connection $\Gamma_{\mu \nu}^{\alpha}$ to vanish identically. Moreover, the parallelization vector fields define a metric tensor on $M$ by

$$
g_{\mu \nu} \equiv \eta_{a b} h_{\mu}^{a} h_{\nu}^{b}
$$

with inverse metric

$$
g^{\mu \nu}=\eta^{a b} h_{a}{ }^{\mu} h_{b}{ }^{\nu}
$$

The Levi-Civita connection associated with $g_{\mu \nu}$ is

$$
\stackrel{\circ}{\Gamma}_{\mu \nu}^{\alpha}=\frac{1}{2} g^{\alpha \sigma}\left(\partial_{\nu} g_{\mu \sigma}+\partial_{\mu} g_{v \sigma}-\partial_{\sigma} g_{\mu \nu}\right) .
$$

In view of (9), the canonical connection $\Gamma^{\alpha}{ }_{\mu \nu}(8)$ is metric:

$$
\nabla_{\sigma}^{(\Gamma)} g_{\mu v} \equiv 0
$$

The torsion tensor of the canonical connection (8) is defined as

$$
T_{\mu \nu}^{\alpha} \equiv \Gamma^{\alpha}{ }_{\nu \mu}-\Gamma_{\mu \nu}^{\alpha}=h_{a}^{\alpha}\left(\partial_{\mu} h^{a}{ }_{\nu}-\partial_{\nu} h_{\mu}^{a}\right)
$$

The contortion tensor $K_{\mu \nu}^{\alpha}$ is defined by

$$
K_{\mu \nu}^{\alpha} \equiv \Gamma_{\mu \nu}^{\alpha}-\stackrel{\circ}{\Gamma}_{\mu \nu}^{\alpha}=h_{a}{ }^{\alpha} \nabla_{\nu}^{(\stackrel{\circ}{\Gamma})} h^{a}{ }_{\mu}
$$

where the covariant derivative $\nabla_{\sigma}^{(\stackrel{\circ}{\Gamma})}$ is with respect to the LeviCivita connection. Since $\stackrel{\circ}{\Gamma}_{\mu \nu}^{\alpha}$ is symmetric, it follows that (using (14)) one can also show the following useful relations:

$$
T_{\alpha \mu \nu}=K_{\alpha \mu \nu}-K_{\alpha \nu \mu}
$$

$$
K_{\alpha \mu \nu}=\frac{1}{2}\left(T_{v \alpha \mu}+T_{\alpha \mu \nu}-T_{\mu \alpha v}\right)
$$

where $T_{\mu \nu \sigma}=g_{\epsilon \mu} T_{\nu \sigma}^{\epsilon}$ and $K_{\mu \nu \sigma}=g_{\epsilon \mu} K_{\nu \sigma}^{\epsilon}$. It is to be noted that $T_{\mu \nu \sigma}$ is skew-symmetric in the last pair of indices whereas $K_{\mu v \sigma}$ is skew-symmetric in the first pair of indices. Moreover, it follows from (15) and (16) that the torsion tensor vanishes if and only if the contortion tensor vanishes. In the teleparallel space there are three Weitzenböck invariants: $I_{1}=T^{\alpha \mu \nu} T_{\alpha \mu \nu}$, $I_{2}=T^{\alpha \mu \nu} T_{\mu \alpha v}$ and $I_{3}=T^{\alpha} T_{\alpha}$, where $T^{\alpha}=T_{\rho}{ }^{\alpha \rho}$. We next define the invariant $T=A I_{1}+B I_{2}+C I_{3}$, where $A, B$ and $C$ are arbitrary constants [4]. For the values: $A=1 / 4, B=$ $1 / 2$ and $C=-1$ the invariant $T$ is just the Ricci scalar up to a total derivative term; then a teleparallel version of gravity equivalent to GR can be achieved [5]. The teleparallel torsion scalar is given in the compact form

$$
T \equiv T^{\alpha}{ }_{\mu \nu} S_{\alpha}{ }^{\mu \nu},
$$

where the superpotential tensor

$$
S_{\alpha}{ }^{\mu \nu}=\frac{1}{2}\left(K_{\alpha}^{\mu v}+\delta_{\alpha}^{\mu} T_{\beta}^{\beta v}-\delta_{\alpha}^{v} T_{\beta}^{\beta \mu}\right),
$$

is skew symmetric in the last pair of indices.

\section{B. $f(T)$ gravity}

There are different extensions of TEGR, e.g. Born-Infeld extension of the TEGR [6,7], another interesting variant is the modified teleparallel equivalent of Gauss-Bonnet gravity and its applications [8-10]. The extension to $f(T)$-gravity has been inspired by the $f(R)$-gravity by replacing the Ricci scalar in the Einstein-Hilbert action instead of the Ricci scalar. But the former is by replacing the teleparallel torsion scalar by an arbitrary function $f(T)$ [11-14]. We consider the action of the $f(T)$-gravity

$$
\mathcal{S}=\int d^{4} x|h|\left[\frac{1}{2 \kappa^{2}} f(T)+L_{m}\right]
$$

where $L_{m}$ is the lagrangian of the matter and $|h|=\sqrt{-g}=$ $\operatorname{det}\left(h_{\mu}{ }^{a}\right)$. The variation of the action (19) with respect to the tetrad gives

$\frac{1}{h} \partial_{\mu}\left(h S_{a}^{\mu \nu}\right) f_{T}-h_{a}^{\lambda} T_{\mu \lambda}^{\rho} S_{\rho}^{v \mu} f_{T}+S_{a}^{\mu \nu} \partial_{\mu} T f_{T T}+\frac{1}{4} h_{a}^{v} f=\frac{\kappa^{2}}{2} h_{a}^{\rho} \mathfrak{I}_{\rho}^{v}$,

where $f=f(T), f_{T}=\frac{\partial f(T)}{\partial T}, f_{T T}=\frac{\partial^{2} f(T)}{\partial T^{2}}$ such that the TEGR theory is recovered by setting $f(T) \stackrel{=}{=} T$. For more details of $f(T)$-gravity, see the recent review [15].

\section{Modified Friedmann equations}

We take the metric to be of the at Friedmann-RobertsonWalker (FRW) form (1). Therefore, the vierbein may take the diagonal form

$$
e_{\mu}{ }^{a}=\operatorname{diag}(1, a(t), a(t), a(t)) .
$$


This directly relates the teleparallel torsion scalar (17) to Hubble parameter as

$$
T=-6 H^{2},
$$

where $H \equiv \dot{a} / a$ is Hubble parameter and we use the dot to denote the differentiation with respect to the cosmic time $t$. Where $\mathfrak{I}_{\mu}{ }^{v}$ is the usual energy-momentum tensor of matter fields. We consider a perfect-fluid material-energy tensor (3). Inserting the vierbein (21) into the field equations (20) for the matter fluid (3), the modified Friedmann equations of the $f(T)$-gravity read

$$
\begin{aligned}
\rho_{m} & =\frac{1}{2 \kappa^{2}}\left[f(T)+24 H^{2} f_{T}\right] \\
p_{m} & =\frac{-1}{2 \kappa^{2}}\left[f(T)+4\left(3 H^{2}+\dot{H}\right) f_{T}-48 \dot{H} H^{2} f_{T T}\right] .
\end{aligned}
$$

In the above, the usual Friedmann equations are recovered by setting $f(T)=T$. Assuming that the matter fluid is governed by the linear equation-of-state $p_{m}=\omega_{m} \rho_{m}$, the system acquires the conservation (continuity) equation

$$
\dot{\rho}_{m}+3 H\left(1+\omega_{m}\right) \rho_{m}=0
$$

\section{Dynamical view of $f(T)$}

We argue that the modified Friedmann equations of any $f(T)$-theory can be viewed as a one-dimensional autonomous system, i.e. $\dot{H}=\mathcal{F}(H)$, if we use the linear equation-of-state of the universe matter. As a consequence we can interpret this differential equation as a vector field on a line introducing one of the basic techniques of dynamics. In order to prove our argument we use the useful relation (22), then the field equations (23) and (24) in flat space can be rewritten in terms of Hubble parameter as [16]

$$
\begin{aligned}
\rho_{m} & =\frac{1}{2 \kappa^{2}}\left[f(H)-H f_{H}\right], \\
p_{m} & =\frac{-1}{2 \kappa^{2}}\left[f(H)-H f_{H}-\frac{1}{3} \dot{H} f_{H H}\right],
\end{aligned}
$$

where $f_{H}:=\frac{d f}{d H}$ and $f_{H H}:=\frac{d^{2} f}{d H^{2}}$. Constraining the modified Friedmann equations to the linear equation-of-state $p_{m}=$ $\omega_{m} \rho_{m}$, we write [16]

$$
\dot{H}=3\left(1+\omega_{m}\right)\left[\frac{f(H)-H f_{H}}{f_{H H}}\right]=\mathcal{F}(H) .
$$

Since $f(H), f_{H}$ and $f_{H H}$ are functions of $H$ only, hence for any $f(T)$-theory we can always write the modified Friedmann equations in flat space as a one-dimensional autonomous system. In order to fix our notations, we follow [17] calling equation (28) the $f(T)$ phase portrait, while its solution $H(t)$ is the phase trajectory. Thus the phase portrait corresponds to any $f(T)$-theory can be drawn in an $(\dot{H}-H)$ phase-space of the Friedmann's system. In this space each point is a phase point and could serve as an initial condition. It is clear that when
$f(H)=-6 H^{2}$, equation (28) reproduces the standard general relativistic phase portrait, i.e.

$$
\dot{H}=-\frac{3}{2}\left(1+\omega_{m}\right) H^{2} .
$$

The last equation can be represented geometrically as shown in Figure 1.

One of the modified gravity theories which has been used widely in cosmology is the $f(T)$ theory. Although, this can be applied generally in modified gravity, the modified Friedmann equations of any $f(T)$-theory can be viewed as a one dimensional autonomous system [16], i.e. $\dot{H}=\mathcal{F}(H)$. This feature is not available for other modified gravity theories, e.g. $f(R)$, which contain higher derivatives of $H$. In this sense we find that the phase space analysis is more consistent with $f(T)$ cosmology. However, similar models have been investigated, without using the phase space, in Gauss-Bonnet modified gravity [18, 19].

\section{BOUNCING MODELS IN $f(T)$ COSMOLOGY}

To reconstruct a bouncing universe in the framework of $f(T)$ and discuss its physical motivation we must follow the below items:

- Select a bouncing scale factor

- Reconstruct $f(T)$ of the bounce universe

- Draw the phase portrait

- Investigate the thermal evolution (slow roll validity)

- Move to Einstein frame

- Investigate the torsion equation-of-state

- Consider the matter component to be a canonical scalar field

- Evaluate the scalar potential of the $f(T)$ theory

- Investigate the equation-of-state and the energy conditions

Now are going to apply the above procedure to two models:

\section{A. Model 1}

In this model the scale factor is given by

$$
R(t)=\sqrt{\beta^{2} t^{2}+R_{0}^{2}} .
$$

From Eq. (30) we get the Hubble parameter

$$
H(t)=\frac{\beta^{2} t}{\beta^{2} t^{2}+R_{0}^{2}} \Rightarrow t(H)=\frac{\beta \pm \sqrt{\beta^{2}-4 H^{2} R_{0}^{2}}}{\beta H} .
$$


The rate of the Hubble parameter is given by

$$
\dot{H}(t)=\frac{\beta^{2}\left(R_{0}{ }^{2}-\beta^{2} t^{2}\right)}{\beta^{2} t^{2}+R_{0}^{2}} \Rightarrow \dot{H}(H)=-\frac{2 \sqrt{\beta^{2}-4 H^{2} R_{0}^{2}}}{\beta \pm \sqrt{\beta^{2}-4 H^{2} R_{0}^{2}}} .
$$

$$
f(t)=\frac{c_{1} t}{\beta^{2} t^{2}+R_{0}^{2}}+c_{2}\left(\frac{\left(t^{2}(4+3 \omega) \beta^{2}+R_{0}^{2}\right)\left[\operatorname{hypergoem}\left(\left[\frac{1}{2}, \frac{3(1+\omega)}{2}\right],\left[\frac{3}{2}\right],-\frac{\beta^{2} t^{2}}{R_{0}{ }^{2}}\right)\right]}{3 \beta^{2}(1+\omega)\left(\beta^{2} t^{2}+R_{0}^{2}\right)}+\frac{\left(t^{2}\left[\operatorname{hypergoem}\left(\left[\frac{3}{2}, \frac{5+3 \omega}{2}\right],\left[\frac{5}{2}\right],-\frac{\beta^{2} t^{2}}{R_{0}{ }^{2}}\right)\right]\right.}{3 R_{0}{ }^{2}}\right),
$$

from which one can write it in terms of the torsion scalar as

$$
\begin{aligned}
& f(T)=\frac{1}{3 T R_{0}^{2} \beta^{2}(1+\omega)\left(3 \beta^{2} \pm \beta \sqrt{6 T R_{0}^{2}+\beta^{2}}\right)}\left(c_{2}\left[T R_{0}^{2} \beta^{2}+2 \beta^{4} \pm \alpha\left(T R_{0}^{2}-6 \beta^{2}\right) \sqrt{6 T R_{0}^{2}+\beta^{2}}\right](1+\omega)\right. \\
& \times \operatorname{hypergoem}\left(\left[\frac{3}{2}, \frac{5+3 \omega}{2}\right],\left[\frac{5}{2}\right],-\frac{T R_{0}^{2}+3 \beta^{2} \pm \beta \sqrt{6 T R_{0}^{2}+\beta^{2}}}{T R_{0}^{2}}\right)+T R_{0}^{2}\left[3 T R_{0}^{2}(1+\omega) \pm c_{2} \sqrt{6 T R_{0}^{2}+\beta^{2}}(3 \omega+4)+3 \beta^{2}(3 \omega+4)\right. \\
& \left.\left.\times \operatorname{hypergoem}\left(\left[\frac{1}{2}, \frac{3(1+\omega)}{2}\right],\left[\frac{3}{2}\right], \frac{T R_{0}^{2}+3 \beta^{2} \pm \beta \sqrt{6 T R_{0}^{2}+\beta^{2}}}{T R_{0}^{2}}\right) \pm \beta c_{1}(1+\omega) \sqrt{\left( \pm \beta \sqrt{6 T R_{0}^{2}+9 \beta^{2}}-T R_{0}^{2}-3 \beta^{2}\right) T}\right]\right)
\end{aligned}
$$

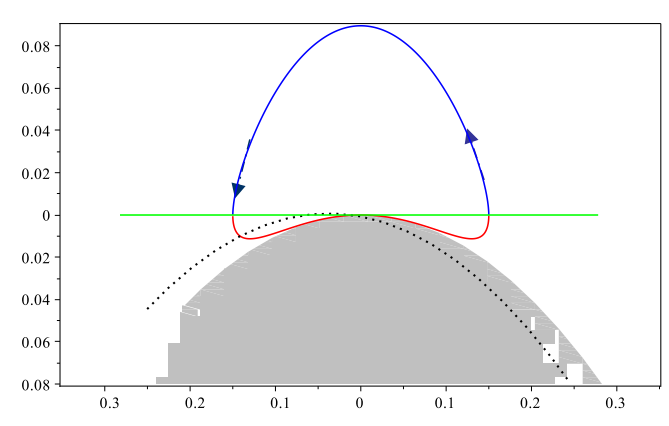

FIG. 5: Phase space of the first model.

It is convenient to transform from the matter frame we have been used to Einstein frame, which gives Einstein's field equations form and additional degrees-of-freedom by $f(T)$-gravity. So we write the modified Friedmann equations in the case of $f(T)$-gravity, i.e.

$$
\begin{aligned}
H^{2} & =\frac{\kappa^{2}}{3}\left(\rho+\rho_{T}\right), \\
2 \dot{H}+3 H^{2} & =-\kappa^{2}\left(p+p_{T}\right),
\end{aligned}
$$

where the standard matter energy density $\rho$ and pressure $p$ have their torsion scalar counterpart while $\rho_{T}$ and $p_{T}$ are defined as,

$$
\begin{gathered}
\rho_{T}=\frac{1}{2 \kappa^{2}}\left[2 T f^{\prime}-f-T\right], \\
p_{T}=\frac{2}{\kappa^{2}} \dot{H}\left(2 T f^{\prime \prime}+f^{\prime}-1\right)-\rho_{T} .
\end{gathered}
$$

are the torsion contributions to the energy density and pressure, respectively, which satisfy the continuity

$$
\dot{\rho}_{T}+3 H\left(\rho_{T}+p_{T}\right)=0 .
$$

One can show that $\rho_{T}$ and $p_{T}$ vanish where $f(T)=T$ and the standard Friedmann equations are recovered. We argue here that the quantities $\rho_{T}$ and $p_{T}$ can explain the early selfacceleration of the universe. Then, by using equations (37) and (38), we can define the effective torsion equation-of-state parameter as 


$$
\begin{aligned}
& \omega_{T} \equiv \frac{p_{T}}{\rho_{T}}=-1+\frac{4 \dot{H}\left(2 T f^{\prime \prime}+f^{\prime}-1\right)}{2 T f^{\prime}-f-T} \\
& =-\frac{1}{3 \beta^{2}\left(t^{2} \beta^{2}-R_{0}^{2}\right)\left[t\left(t^{2} \beta^{2}+R_{0}^{2}\right)^{3} f^{\prime}+\left(t^{2} \beta^{2}-R_{0}^{2}\right)\left\{\left(t^{2} \beta^{2}+R_{0}^{2}\right)^{2} f-6 \beta^{4} t^{2}\right\}\right]}\left[\left(t^{2} \beta^{2}-R_{0}^{2}\right)\left(t^{2} \beta^{2}+R_{0}^{2}\right)^{4} f^{\prime \prime}-\beta^{2}\left\{t\left(t^{2} \beta^{2}+R_{0}{ }^{2}\right)^{3}\right.\right. \\
& \left.\times\left(9 R_{0}{ }^{2}-5 t^{2} \beta^{2}\right) f^{\prime}-3\left(t^{2} \beta^{2}-R_{0}{ }^{2}\right)^{2}\left[\left(t^{2} \beta^{2}+R_{0}^{2}\right)^{2} f-2 \beta^{4} t^{2}-4 R_{0}^{2} \beta^{2}\right]\right] .
\end{aligned}
$$

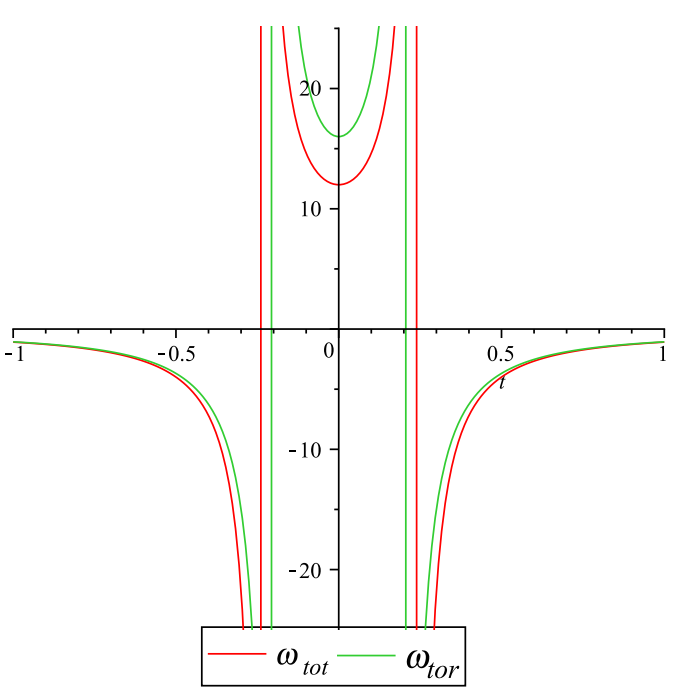

FIG. 6: Eq. of state for total matter and torsion of the first model.

We also define the effective (total) equation-of-state parameter

$$
\omega_{e f f} \equiv \frac{p+p_{T}}{\rho+\rho_{T}}=-\frac{t^{2} \beta^{2}+2 R^{2}}{3 \beta^{2} t^{2}} .
$$

As is clear from Fig. 6 that about bounce time (i.e., $t=0$ ) we have either $\omega_{e f f}>1$ and $\omega_{T}>1$ which are needed for a bounce universe to wash up the anisotropies.

\section{B. Model 2}

In this model the scale factor is given by

$$
R(t)=R_{0}\left(e^{\alpha t}+e^{-\alpha t}\right) .
$$

From Eq. (42) we get the Hubble parameter

$$
H(t)=\frac{\alpha\left(e^{\alpha t}-e^{-\alpha t}\right)}{e^{\alpha t}+e^{-\alpha t}} \Rightarrow t(H)=\frac{\ln \left( \pm \sqrt{\frac{\alpha+H}{\alpha-H}}\right)}{\alpha},
$$

The rate of the Hubble parameter is given by

$$
\dot{H}(t)=\frac{4 \alpha^{2} e^{2 \alpha t}}{\left(1+e^{2 \alpha t}\right)^{2}} \Rightarrow \dot{H}(H)=\alpha^{2}-H^{2} .
$$

Figure 7 shows the phase portrait of the second models which is clearly coincides with GR plus a positive cosmological constant, $\Lambda>0$, where $\Lambda$ in this case is related to the constant $\alpha$.

The form of the function $f$ has the form

$$
\begin{aligned}
& f(t)=\frac{1}{\left(1+e^{2 \alpha t}\right)^{3(1+\omega)}}\left[c_{1} \text { hypergoem }\left(\left[-3(1+\omega), \frac{-(1+3 \omega)}{2}\right],\left[\frac{-3(1+\omega)}{2}\right], e^{2 \alpha}\right)\right. \\
& \left.+c_{2} e^{t \alpha(5+3 \omega)} \text { hypergoem }\left(\left[2, \frac{-(1+3 \omega)}{2}\right],\left[\frac{7+3 \omega}{2}\right],-e^{2 \alpha}\right)\right],
\end{aligned}
$$

where hypergoem $(\mathrm{n}, \mathrm{d}, \mathrm{z})$ is the generalized hypergeometric function $F(n, d, z)$. One can write Eq. (33) in terms of the torsion scalar in the form

$$
\begin{aligned}
& f(T)=\left(T+6 \alpha^{2}\right)^{\frac{5+3 \omega}{4}}\left[c_{1} \operatorname{Leg} P\left(\frac{3 \omega+1}{2}, \frac{3 \omega+5}{2}, \frac{\sqrt{-T}}{6 \alpha}\right)\right. \\
& \left.+c_{2} \operatorname{Leg} Q\left(\frac{3 \omega+1}{2}, \frac{3 \omega+5}{2}, \frac{\sqrt{-T}}{6 \alpha}\right)\right]
\end{aligned}
$$

where $\operatorname{Leg} P(v, x)$ and $\operatorname{Leg} Q(v, x)$ are the Legendre functions of the first and second kinds respectively. 


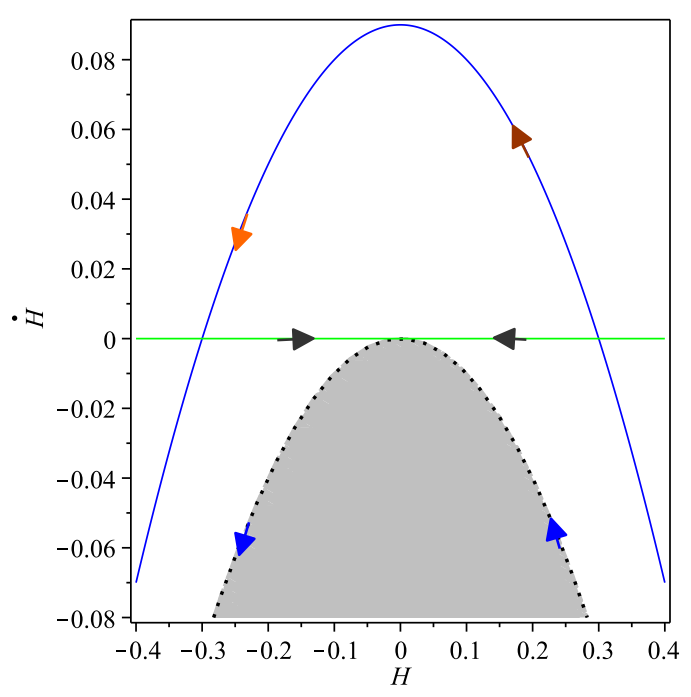

FIG. 7: Phase space of the second model.

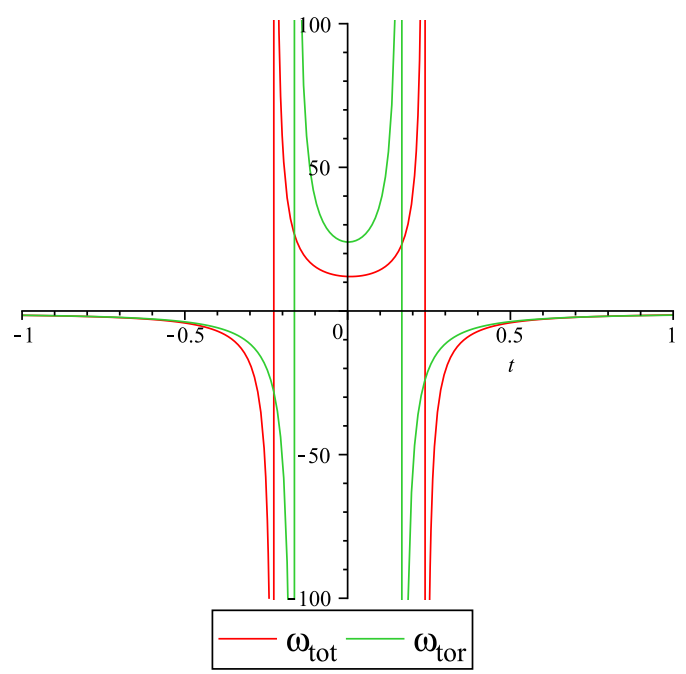

FIG. 8: Phase space of the second model.

Figure 8 shows that about bounce time (i.e., $t=0$ ) we have both $\omega_{\text {eff }}>1$ and $\omega_{T}>1$ as discussed in model 1 .

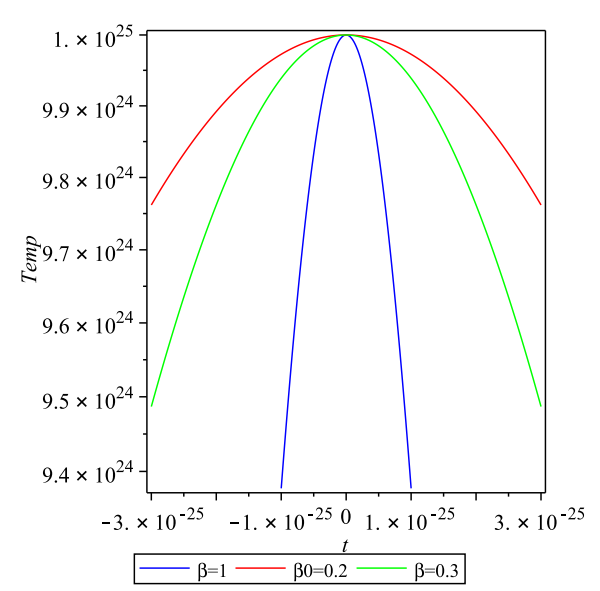

FIG. 9: The temperature of the first model

\section{THERMALIZATION OF THE UNIVERSE}

According to the first law of thermodynamics, in the expanding universe, we have

$$
\Theta d S=d(\rho V)+p d V
$$

with the integrability condition [20]

$$
\frac{\partial^{2} S}{\partial \Theta \partial V}=\frac{\partial^{2} S}{\partial V \partial \Theta},
$$

the energy density and pressure satisfy

$$
\frac{d p}{d \Theta}=\frac{\rho+p}{\Theta} .
$$

Using (47) and (48), we evaluate the temperature

$$
\begin{aligned}
\Theta(t) & =\Theta_{0} e^{\int \frac{\frac{d}{d t} p(t)}{\rho(t)+p(t)} d t}, \\
& \propto a^{-3 \omega}=\Theta_{0} a^{-3 \omega},
\end{aligned}
$$

where $\Theta_{0}=\Theta\left(t_{0}\right)$ is an arbitrary constant, with a dimension $\mathrm{K}$. We assume the boundary condition so that the temperature $\Theta \sim 2.74 \mathrm{~K}$ at the present time where $t_{0} \sim 10^{17} \mathrm{sec}$. Using Eq. (49) we plot the temperature of the first and second models as shown in Figs. 9 and 10. As shown in Fig 9. that the temperature depends on the parameter $\beta$. In Fig. 10 it is clear that in early time the temperature is low which is a good environment to slow roll condition. Therefore, we are going to study the matter content as a scalar field in the next section. 


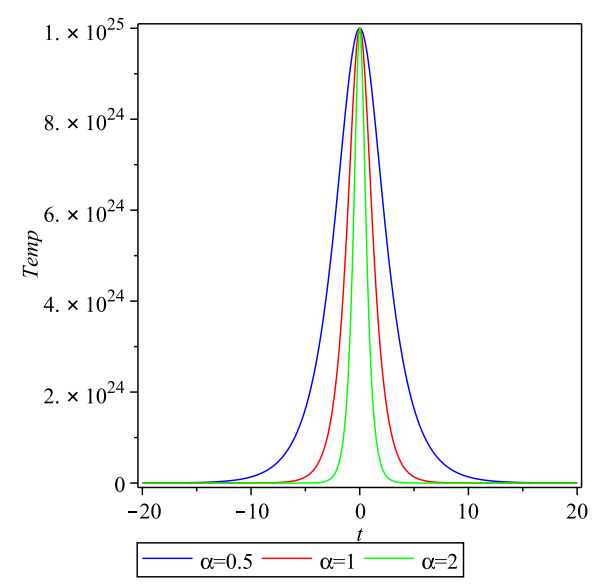

FIG. 10: The temperature of the second model

\section{MATTER AS A CANONICAL SCALAR FIELD}

As is well known that standard inflationary models assume a scalar field (inflaton) $\varphi$, where its density and pressure are defined by

$$
\begin{aligned}
& \rho_{\varphi}=\frac{\dot{\varphi}^{2}}{2}+V(\varphi), \\
& p_{\varphi}=\frac{\dot{\varphi}^{2}}{2}-V(\varphi) .
\end{aligned}
$$

Where $\dot{\varphi}^{2}$ represents a kinetic term of the inflaton and $V(\varphi)$ is its potential. Accordingly, the inflaton equation-of-state parameter $\omega_{\varphi}:=p_{\varphi} / \rho_{\varphi} \cong-1$, where $V(\varphi) \gg \dot{\varphi}^{2}$. This assumption (slow-roll) can not be justified unless the temperature at this episode is very law.

Combining (50), (51), (35) and (36), we write the kinetic and the potential of the scalar field

$$
\begin{gathered}
\dot{\phi}^{2}(t)=\frac{1}{6 \kappa^{2}}\left(\frac{\dot{H} \ddot{f}-\dot{f} \ddot{H}}{\dot{H}^{2}}\right), \\
V(t)=\frac{1}{2 \kappa^{2}}\left[f+\left(\frac{\ddot{H}}{6 \dot{H}^{2}}-\frac{H}{\dot{H}}\right) \dot{f}-\frac{\ddot{f}}{6 \dot{H}}\right] .
\end{gathered}
$$

In order to be consistent with literature we may use $\kappa^{2}=1 / M_{p}^{2}$ with $M_{p}=1.22 \times 10^{19} \mathrm{GeV}$. The above equations are consistent with the scalar field background (Klein-Gordon) equation of homogeneous scalar field in the expanding FRW universe

$$
\ddot{\phi}+3 H \dot{\phi}+\frac{d V}{d \phi}=0
$$

\section{A. Model 1}

Using Eqs. (52) - (54) we get

$$
\dot{\phi}^{2}=\frac{-c_{2}}{6 \alpha^{2} \kappa^{2} R_{0}^{2}}\left[\alpha^{2} t^{2}(1+\omega) \times \text { hypergeom }\left(\left[\frac{3}{2}, \frac{5+3 \omega}{2}\right],\left[\frac{5}{2}\right], \frac{-\alpha^{2} t^{2}}{R_{0}^{2}}\right)-\text { hypergeom }\left(\left[\frac{1}{2}, \frac{3(1+\omega)}{2}\right],\left[\frac{3}{2}\right], \frac{-\alpha^{2} t^{2}}{R_{0}^{2}}\right) R_{0}^{2}\right],
$$

and the potential has the form

$$
\begin{aligned}
& v(t)=\frac{-3 c_{2}}{28 \alpha^{2} \kappa^{2} R_{0}{ }^{6}(1+\omega)\left(\alpha^{2} t^{2}-R_{0}{ }^{2}\right)^{2}}\left[-7 / 5 R_{0}^{2}(1+\omega) t^{2}\left([17 / 3+\omega] \alpha^{4} t^{4}-[5+\omega] \alpha^{2} t^{2} R_{0}^{2}-2 R_{0}^{4}\right)[5 / 3+\omega]\left[\alpha^{2} t^{2}+R_{0}^{2}\right]\right. \\
& \times \text { hypergeom }\left(\left[\frac{5}{2}, \frac{7+3 \omega}{2}\right],\left[\frac{7}{2}\right], \frac{-\alpha^{2} t^{2}}{R_{0}^{2}}\right)+(1+\omega) t^{4}\left(\alpha^{2} t^{2}-R_{0}{ }^{2}\right) \alpha^{4}[7 / 3+\omega]\left(\beta^{2} t^{2}+R_{0}^{2}\right)^{2}(5 / 3+\omega) \\
& \times \text { hypergeom }\left(\left[\frac{7}{2}, \frac{3(3+\omega)}{2}\right],\left[\frac{9}{2}\right], \frac{-\alpha^{2} t^{2}}{R_{0}{ }^{2}}\right)+7 R_{0}^{4}(1+\omega)\left([20 / 9+\omega] \alpha^{6} t^{6}-8 / 9[15 / 8+\omega] \alpha^{4} t^{4} R_{0}^{2}\right. \\
& \left.-5 / 9 R_{0}{ }^{4} t^{2} \alpha^{2}(16 / 5+\omega)-1 / 9 R_{0}{ }^{6}\right) \times \text { hypergeom }\left(\left[\frac{3}{2}, \frac{5+3 \omega}{2}\right],\left[\frac{5}{2}\right], \frac{-\alpha^{2} t^{2}}{R_{0}^{2}}\right)-14 / 3 R_{0}^{6}\left([4 / 3+\omega] \alpha^{4} t^{4}\right. \\
& \left.\left.-4 / 3[3 / 2+\omega] \alpha^{2} t^{2} R_{0}^{2}-1 / 3 R_{0}{ }^{4} \omega\right) \times \text { hypergeom }\left(\left[\frac{1}{2}, \frac{3(1+\omega)}{2}\right],\left[\frac{3}{2}\right], \frac{-\alpha^{2} t^{2}}{R_{0}{ }^{2}}\right)\right]+V_{0}
\end{aligned}
$$

where $c_{2}$ and $V_{0}$ are constants of integration.

By the same method one can calculate the scalar field $\phi$ and the potential of the second model. Figures 10-14 shows 


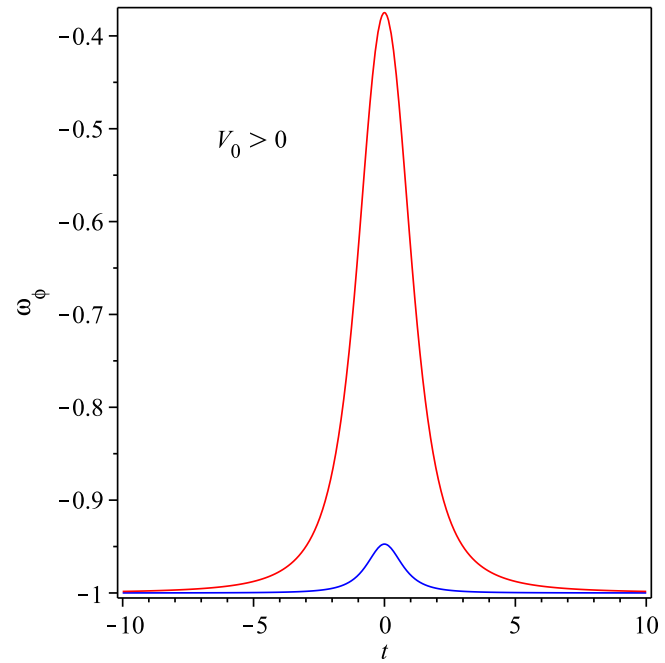

FIG. 11: The Eq. of state of the scalar field of the first model model

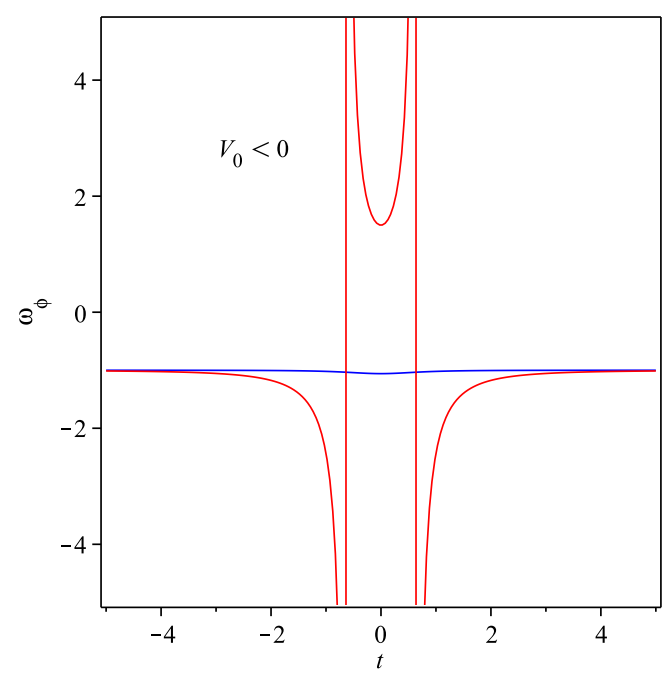

FIG. 12: The Eq. of state of the scalar field of the first model model

the behaviors of the equation of state of the scalar field for positive and negative quantities of the constant of integration $V_{0}$ when the parameter $\omega=0$.

\section{B. Model 2}

\section{Energy conditions}

In this subsection we investigate another major problem that usually faces bouncing models, that is the violation of the NEC, which gives rise to ghost instability problem. In the case of a perfect fluid, these energy conditions strong energy condition, SEC, and null energy condition ,NEC, must satisfy $\rho+p \geq 0$ and $\rho+3 p \geq 0$, while the weak energy condition, WEC, and dominant energy condition, DEC, demand the fol-

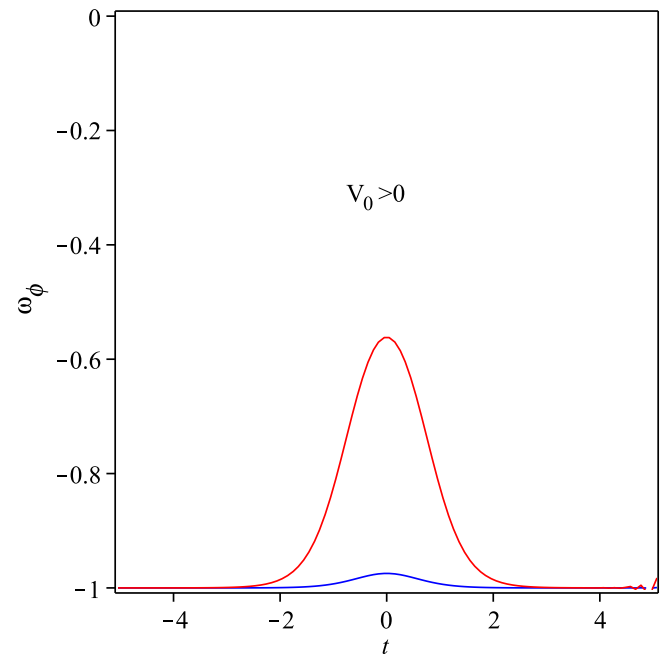

FIG. 13: The Eq. of state of the scalar field of the second model model

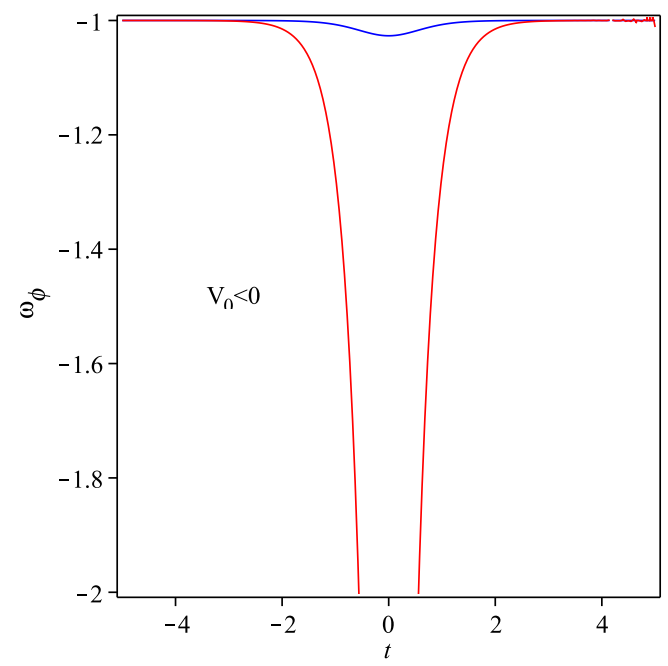

FIG. 14: The Eq. of state of the scalar field of the second model model

lowing constrains $\rho \geq 0$ and $\rho \pm p \geq 0$.

We summaries the energy conditions of the perfect fluid as

$$
\begin{array}{lc}
\text { Name } & \text { For perfect fluid } \\
\text { Weak } & \rho_{\phi} \geq 0, \quad \rho_{\phi}+p_{\phi} \geq 0 ; \\
\text { Null } & \rho_{\phi}+p_{\phi} \geq 0 ; \\
\text { Strong } & \rho_{\phi}+p_{\phi} \geq 0, \quad \rho_{\phi}+3 p_{\phi} \geq 0 ; \\
\text { Dominant } & \rho_{\phi} \geq\left|p_{\phi}\right| .
\end{array}
$$

Using the above conditions we plot the energy conditions of the first and second models in figures 15 and 16. As clear from these figures that the four energy conditions are fulfilled. 


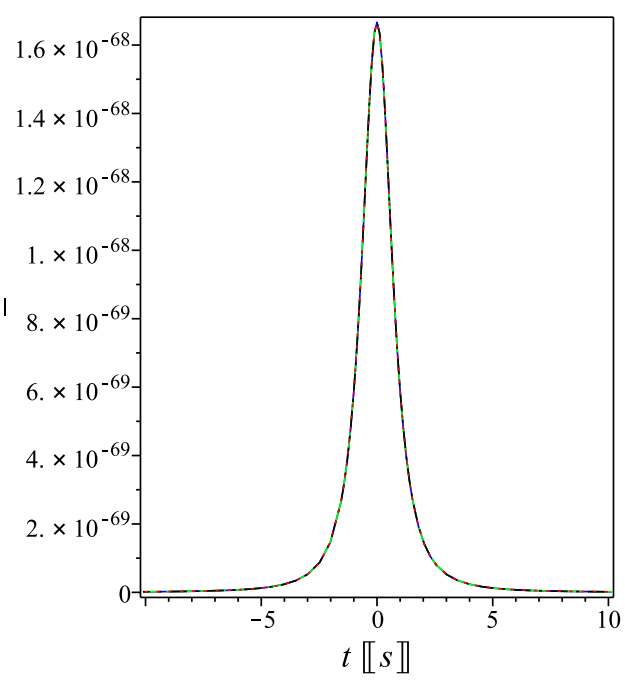

FIG. 15: The energy conditions of the first model model

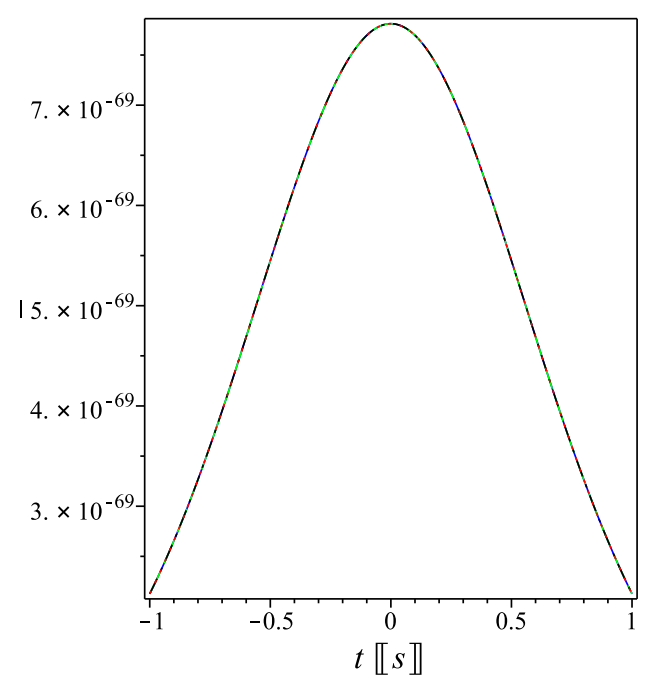

FIG. 16: The energy conditions of the second model model

\section{CONCLUSION}

The results of this study can be summarized as:

- The GR applications in cosmology are very limited.

- The $f(T)$-theories have more flexibility to overcome the limitation of GR.

- The thermal evolution in general has the form $\Theta \propto a^{-3 \omega}$. Figure (10) shows that at the early time the temperature is low and this is a good environment to slow roll conditions.
- The torsion gravity provides a good candidate to describe the bounce behavior at early time (to wash the anisotropies).

- The canonical scalar field matter in $f(T)$ does not violate the NEC.

\section{REFERENCES}

1. N. Breton, J.L. Cervantes-Cota, and M. Salgad, eds., The Early Universe and Observational Cosmology, vol. 646 of Lecture Notes in Physics, Berlin Springer Verlag (2004).

2. A. Awad, Physical Review D 87, 103001 (2013), 1303.2014.

3. S. Nojiri and S. D. Odintsov, Physics Reports 505, 59 (2011), 1011.0544.

4. J.W. Maluf, Annalen der Physik 525, 339 (2013), arXiv: gr-qc/1303.3897, 1303.3897.

5. G.G.L. Nashed, Astrophysics \& Space Science 330, 173 (2010), 1503.01379.

6. R. Ferraro and F. Fiorini, Physical Review D 78, 124019 (2008), 0812.1981.

7. F. Fiorini, Physical Review Letters 111, 041104 (2013), 1306.4392 .

8. G. Kofinas and E. Saridakis, Physical Review D 90, 084044 (2014), 1404.2249.

9. G. Kofinas and E. Saridakis, Physical Review D 90, 084045 (2014), 1408.0107.

10. G. Kofinas, G. Leon, and E. Saridakis, Classical and Quantum Gravity 31, 175011 (2014), 1404.7100.

11. G. R. Bengochea and R. Ferraro, Physical Review D 79, 124019 (2009), 0812.1205.

12. G. G. L. Nashed and W. El Hanafy, European Physical Journal C 74, 3099 (2014), 1403.0913.

13. G. G. L. Nashed, Chin. Phys. Lett. 29, 050402 (2011), 1111.0003.

14. E. V. Linder, Physical Review D 81, 127301 (2010), 1005.3039.

15. Y.-F. Cai, S. Capozziello, M. De Laurentis, and E. N. Saridakis (2015), 1511.07586.

16. A. Awad, W. El Hanafy, G. G. L. Nashed, and E. N. Saridakis (2016), in progress.

17. S. H. Strogatz, Nonlinear Dynamics And Chaos: With Applications To Physics, Biology, Chemistry And Engineering, vol. 1 of Studies in Nonlinearity (1994), ISBN 0-20154344-3,9780201543445.

18. V. K. Oikonomou, Phys. Rev. D 92, 124027 (2015), 1509.05827.

19. V. K. Oikonomou, Astrophysics and Space Science 361, 1 (2016), ISSN 1572-946X.

20. S. Weinberg, Gravitation and cosmology: principles and applications of the general theory of relativity (Wiley, 1972), ISBN 9780471925675. 
Нашед Дж.; 2; 3 и Ханафи В. Эль ${ }^{1 ; 3}$

${ }^{1}$ Центр теоретической физики,

Британский университет в Египте

${ }^{2}$ Кафедра математики, факультет наук,

Университет Айн Шамс

${ }^{3}$ Египетская группа относительности

Нашед Дж., Ханафи В. Эль

Построение $f(T)$-теории из колеблющейся

Вселенной Фридмана

Показано, что телепараллельная $f(\mathrm{~T})$-гравитация может предоставить совместимые версии космологии колебаний. В данной работе были изучены две модели колеблющихся вселенных, описаны их фазовые портреты посредством построения моделей фазового пространства. Также обсуждается процесс термализации Вселенной во время периода колебаний. Заново написаны решения в рамках теории Эйнштейна с целью описания гравитации кручения как дополнительной степени свободы. Изучена эволюция уравнения состояния кручения жидкостей. Допуская, что исследуемый компонент является каноническим скалярным полем, получены потенциалы областей скалярного поля, вызванные $f(\mathrm{~T})$-гравитацией двух предложенных моделей. Исследована эволюция уравнения состояния скалярного поля во время периода колебания. Дополнительно были изучены энергетические условия двух моделей. В заключение, обсуждается обоснованность медленного вращения в космологии колебаний.

Ключевые слова: инфляция, скалярное поле, телепераллелизм. 